\title{
A review of specialties performing temporal artery biopsies in Ontario: a retrospective cohort study
}

\author{
Jonathan A. Micieli MD CM, Robert Micieli BSc, Edward A. Margolin MD
}

Abstract

Background: Temporal artery biopsy is the gold standard for the diagnosis of giant cell arteritis, but the numbers and types of surgical specialists performing temporal artery biopsies are unknown. The goal of this study was to determine which surgical specialists performed temporal artery biopsies and how geographic location influenced this trend over a period of 10 years.

Methods: This retrospective cohort study included all physicians practising in Ontario from 2002 to 2013 . Using comprehensive physician services data from the IntelliHEALTH Medical Services database, physicians performing temporal artery biopsies were identified by the Ontario Health Insurance Plan billing code submitted for remuneration. Physicians were categorized by specialty and geographic Local Health Integration Unit.

Results: A total of 9958 patients underwent a temporal artery biopsy during the study period: the biopsies were performed by 11 different subspecialties. The number of patients undergoing a temporal artery biopsy declined over the 10-year study period. Most procedures were performed by general surgeons (38.1\%), followed by ophthalmologists $(31.0 \%)$ and plastic surgeons (23.6\%). Ophthalmologists performed more temporal artery biopsies per person compared with general surgeons, but significantly more general surgeons performed at least 1 biopsy. There was significant variation based on geographic location: plastic surgeons performed the most biopsies in regions with a population of more than 1 million people, and general surgeons performed most of the biopsies in rural areas.

Interpretation: Geographic location heavily influenced which specialty was most likely to perform temporal artery biopsies. General surgery, ophthalmology and plastic surgery emerged as leaders in this area, and their residency programs should include formal training in this procedure in their curriculua.

iant cell arteritis, also known as temporal arteritis, is an inflammatory vasculopathy of unknown cause that occurs in people over 50 years of age. Typically, it involves the branches of the external carotid artery and the vertebral, ophthalmic and posterior ciliary arteries. Biopsy of the temporal artery is considered the gold standard in the diagnosis of giant cell arteritis, because it provides clinicians with the rationale to use high-dose corticosteroids for prolonged periods of time and avoids unnecessary treatment in patients who do not actually have the disease. ${ }^{1}$ Generally, patients are referred to a surgical specialist for a temporal artery biopsy, but the frequency with which different specialties perform temporal artery biopsies is unknown. This information is important for determining which surgical residency training programs should ensure competency in this area and for identifying the specialists who can be referred patients by medical physicians such as rheumatologists and neurologists. Although it is a generally safe procedure, temporal artery biopsy still carries a small risk of complications including scalp necrosis, ${ }^{2}$ facial nerve injury ${ }^{3,4}$ and stroke, ${ }^{5}$ and proper training and procedure volume during residency are important to avoid unnecessary complications in practice.
The goal of the current study was to determine which surgical specialists perform temporal artery biopsies and how this has evolved over a 10-year period. The influence of geographic location and urban versus rural setting were also studied in relation to procedure output. Population-level data addressing this issue were lacking.

\section{Methods}

\section{Design and setting}

This was a retrospective cohort study in Ontario, which is Canada's most populous province and had a population that ranged from 7.2 to 9.4 million people who were over 50 years of age during the study period. Ontario provides universal government-funded health care to all of its citizens through

Competing interests: None declared.

This article has been peer reviewed.

Correspondence to: Jonathan Micieli, jonathan.micieli@utoronto.ca

CMAJ Open 2015. DOI:10.9778/cmajo.20140108 
the Ontario Health Insurance Plan. Licensed physicians in Ontario receive fee-for-service payment by submitting specific billing codes; they cannot bill patients directly for services covered by the Ontario Health Insurance Plan. This means that the Ontario population is an ideal population for the study of procedure output. The Ontario Health Insurance Plan billing database accessed through the IntelliHEALTH ONTARIO system was used in previous population-based studies. ${ }^{6,7}$ Previous studies showed an over $90 \%$ agreement between physician claims and clinical records. ${ }^{8}$ Ethics approval for this study was obtained from the Mount Sinai Hospital Research Ethics Board.

\section{Sources of data}

The OHIP billing codes were accessed via the Medical Services database in the IntelliHEALTH ONTARIO system, which contains detailed information on physician billing, procedures and hospital information in the province. The Ontario Schedule of Benefits published each year by the Ministry of Health and Long-Term Care contains the specific billing codes that physicians must use for each procedure and the financial remuneration for each service. All physicians who perform temporal artery biopsies must submit the specific code Z815A to receive remuneration for the procedure. The Medical Services database classifies each physician by his or her subspecialty, which is based on the specialty that they billed the most during a fiscal year. Data for each specialist or general physician who submitted the Z815A code for the fiscal years 2003/12 (Apr. 1, 2003, to Mar. 31, 2013) were retrieved and studied. Bilateral biopsies were not considered in the study because the number of patients undergoing temporal artery biopsy was used as the output filter.

To determine the regional variation in temporal artery biopsies, the procedure output per Local Health Integration Unit was calculated. The province of Ontario is divided into 14 different Local Health Integration Units based on geogra-

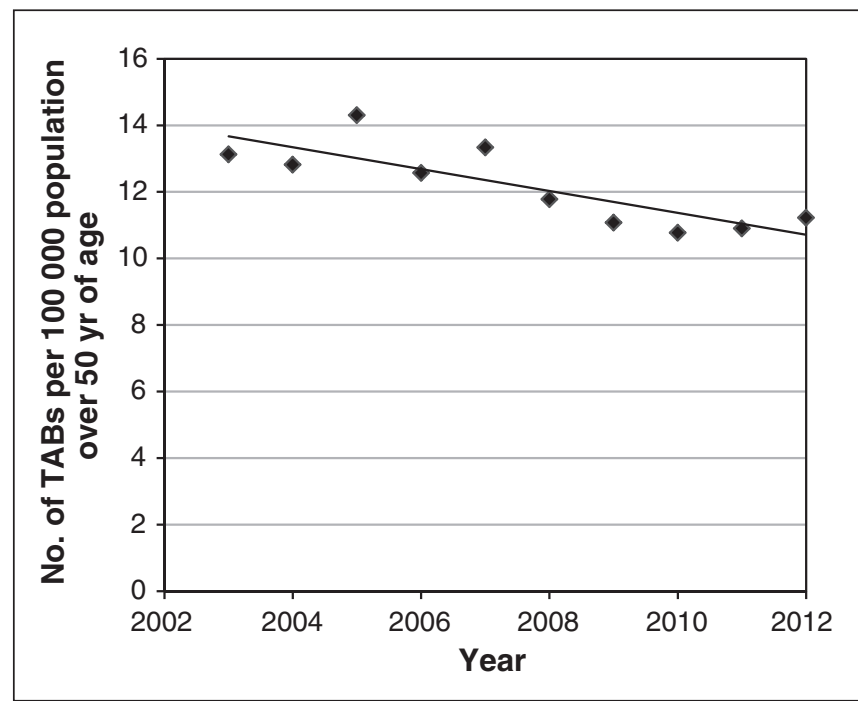

Figure 1: Number of patients undergoing temporal artery biopsies (TABs) per year. Linear line of best fit was plotted for the number of TAB cases (linear regression, $y=-0.33 x+672.6, R^{2}=0.6751$ ). phy: each Local Health Integration Unit plans, integrates and funds local health care. There are 5 different Local Health Integration Units that include ophthalmology residency programs: Toronto Central (at University of Toronto), Hamilton Niagara Haldimand Brant (at McMaster University, Hamilton), Champlain (at University of Ottawa), South East (at Queen's University, Kingston) and South West (at Western University, London). To determine if there was variation in the type of specialists performing temporal artery biopsies based on population size, Local Health Integration Units were divided into those with fewer than 600000 people (i.e., North West, North Simcoe Muskoka, South East and North East), those with 600000 to 1 million people (i.e., Erie St. Clair, Waterloo Wellington, Central West and South West) and those with more than 1 million people (i.e., Toronto Central, Mississauga Halton, Champlain, Hamilton, Central East and Central). This category was based on a previous study assessing the distribution of ophthalmologists in Ontario. ${ }^{6}$

\section{Statistical methods}

The IntelliHEALTH ONTARIO data were exported to SAS version 9.4 and analyzed statistically. A linear regression was used to explore the relationship between the number of temporal biopsies and year. Because the dataset included all physicians in Ontario who performed a temporal artery biopsy, no statistical tests for comparing mean number of biopsies per year were required.

\section{Results}

The number of patients over 50 years of age undergoing temporal artery biopsy per year in Ontario declined over the 10 -year study period when standardized for the Ontario population over 50 years of age (linear regression, $y=-0.33 x+$ $672.6, R^{2}=0.6751$ (Figure 1). Eleven different subspecialists performed at least 1 biopsy during the study period (i.e., oph-

\begin{tabular}{|lr|}
\hline $\begin{array}{l}\text { Table 1: Temporal artery biopsies (TABs) performed } \\
(\boldsymbol{n}=\mathbf{9 9 5 8 )} \text { in Ontario by specialty (2003-2012) }\end{array}$ \\
\hline Physician specialty & $\begin{array}{c}\text { No) of TABs } \\
n=9958\end{array}$ \\
\hline Cardiac surgery & $58(0.6)$ \\
\hline Emergency medicine & $1(0.0)$ \\
\hline Family medicine & $27(0.3)$ \\
\hline General surgery & $3791(38.1)$ \\
\hline Neurosurgery & $66(0.7)$ \\
\hline Ophthalmology & $3088(31.0)$ \\
\hline Otolaryngology & $497(5.0)$ \\
\hline Plastic surgery & $2349(23.6)$ \\
\hline Thoracic surgery & $43(0.4)$ \\
\hline Urology & $21(0.2)$ \\
\hline Vascular surgery & $17(0.2)$ \\
\hline
\end{tabular}


thalmology, plastic surgery, otolaryngology, general surgery, vascular surgery, thoracic surgery, neurosurgery, urology, family medicine, emergency medicine and cardiac surgery). Over the 10-year period, general surgeons as a group performed the most temporal artery biopsies, followed by ophthalmologists and plastic surgeons (Table 1). However, of the physicians who performed temporal artery biopsies, ophthalmologists performed more per person each year compared with general surgeons, neurosurgeons and family physicians. Significantly more general surgeons performed at least 1 temporal artery biopsy compared with ophthalmologists, plastic surgeons and otolaryngologists (Table 2).

Which type of medical specialty that performed the most temporal artery biopsies varied by geographic location. General surgeons performed more temporal artery biopsies per person in less-populated regions (<600 000 people) compared with regions with a larger population $(p<0.01$; Figure 2$)$. Ophthalmologists in regions with a population between 600000 and 1 million people performed more biopsies per person than ophthalmologists in regions with fewer than 600000 or more than 1 million people. Plastic surgeons in areas with more than 1 million people performed more biopsies per person than plastic surgeons in regions with fewer than 600000 people. General surgeons performed the most temporal artery biopsies in less-populated regions, ophthalmologists and general surgeons performed the most in regions with 600000 to 1 million people, and plastic surgeons performed the most biopsies per person in regions with more than 1 million people (Figure 2).

The medical specialists performing temporal artery biopsies also varied significantly in the regions with residency training programs (Figure 3). Ophthalmologists in the Champlain and South West Local Health Integration Units performed most of the biopsies in their respective regions over

Table 2: Mean no. of temporal artery biopsies (TABs) performed in Ontario per specialist per year and mean no. of physicians performing at least 1 TAB each year (2003-2012)

\begin{tabular}{|lcc|}
\hline $\begin{array}{l}\text { Physician } \\
\text { specialty }\end{array}$ & $\begin{array}{c}\text { No. of TABS per } \\
\text { physician per yr, } \\
\text { mean } \pm \text { SD }\end{array}$ & $\begin{array}{c}\text { No. of physicians } \\
\text { performing at least 1 } \\
\text { TAB each yr, } \\
\text { mean } \pm \text { SD }\end{array}$ \\
\hline General surgery & $2.8 \pm 2.8$ & $137.3 \pm 11.3$ \\
\hline Ophthalmology & $9.2 \pm 1.4$ & $33.6 \pm 3.6$ \\
\hline Plastic surgery & $4.5 \pm 4.6$ & $51.8 \pm 4.6$ \\
\hline Otolaryngology & $4.2 \pm 6.4$ & $11.8 \pm 3.8$ \\
\hline Neurosurgery & $1.8 \pm 1.4$ & $3.7 \pm 2.1$ \\
\hline Cardiac surgery & $3.4 \pm 2.6$ & $1.7 \pm 0.9$ \\
\hline Family medicine & $1.4 \pm 0.7$ & $2.0 \pm 1.2$ \\
\hline Thoracic surgery & $2.4 \pm 1.2$ & $1.8 \pm 0.6$ \\
\hline Urology & $2.6 \pm 3.5$ & $0.7 \pm 0.7$ \\
\hline Vascular surgery & $4.3 \pm 4.3$ & $0.4 \pm 0.5$ \\
\hline Note: SD = standard deviation. & \\
\hline
\end{tabular}

the entire 10-year study period. On the contrary, ophthalmologists in Toronto and Hamilton performed only a small proportion of the temporal artery biopsies in their areas. General surgeons performed the most biopsies in the South East Local Health Integration Unit and performed a substantial proportion of the biopsies in the South West and Hamilton Local Health Integration Units but not in Toronto and Champlain. Toronto was the only Local Health Integration Unit in which plastic surgeons were the leading specialty, and plastic surgeons in the other areas with residency training programs performed only a small proportion of the temporal artery biopsies in their area. These results are displayed graphically in Figure 3.

\section{Interpretation}

\section{Main findings}

This study found that the number of temporal artery biopsies performed per 100000 population over 50 years of age declined over a 10-year period, and physicians representing 11 different subspecialties performed at least 1 temporal artery biopsy. Most of the biopsies were performed by general surgeons, ophthalmologists and plastic surgeons. Although more general surgeons performed at least 1 biopsy, ophthalmologists performed significantly more temporal artery biopsies per person. There was significant regional variation in which specialty performed the most temporal artery biopsies: general surgeons conducted most biopsies in areas with smaller populations, and plastic surgeons were the major specialty in the most populated areas.

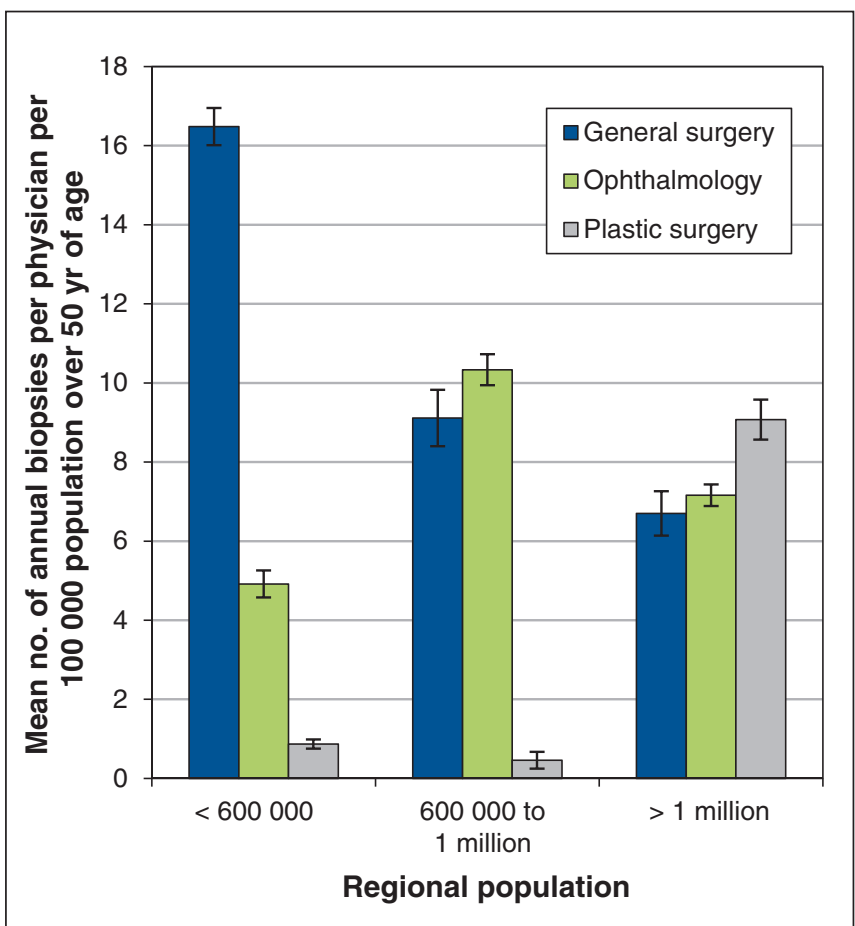

Figure 2: Mean number of temporal artery biopsies performed per specialty group by regional populations of the Local Health Integration Units (LHINs). 


\section{Explanation and comparison to other studies}

At the population level, the number of temporal artery biopsies performed per 100000 population over 50 years of age declined over the 10-year period, which may be accounted for by several factors. First, demographic patterns in the population of Ontario have changed over time, with people at lower risk of giant cell arteritis (e.g., black people, people of Asian or Hispanic descent) accounting for a greater proportion of the population. ${ }^{9-12}$ Second, physicians may be opting out of performing temporal artery biopsies but continuing to treat patients as if they had the disease. A survey of physicians in the United States ${ }^{13}$ found that $15 \%-35 \%$ of physicians

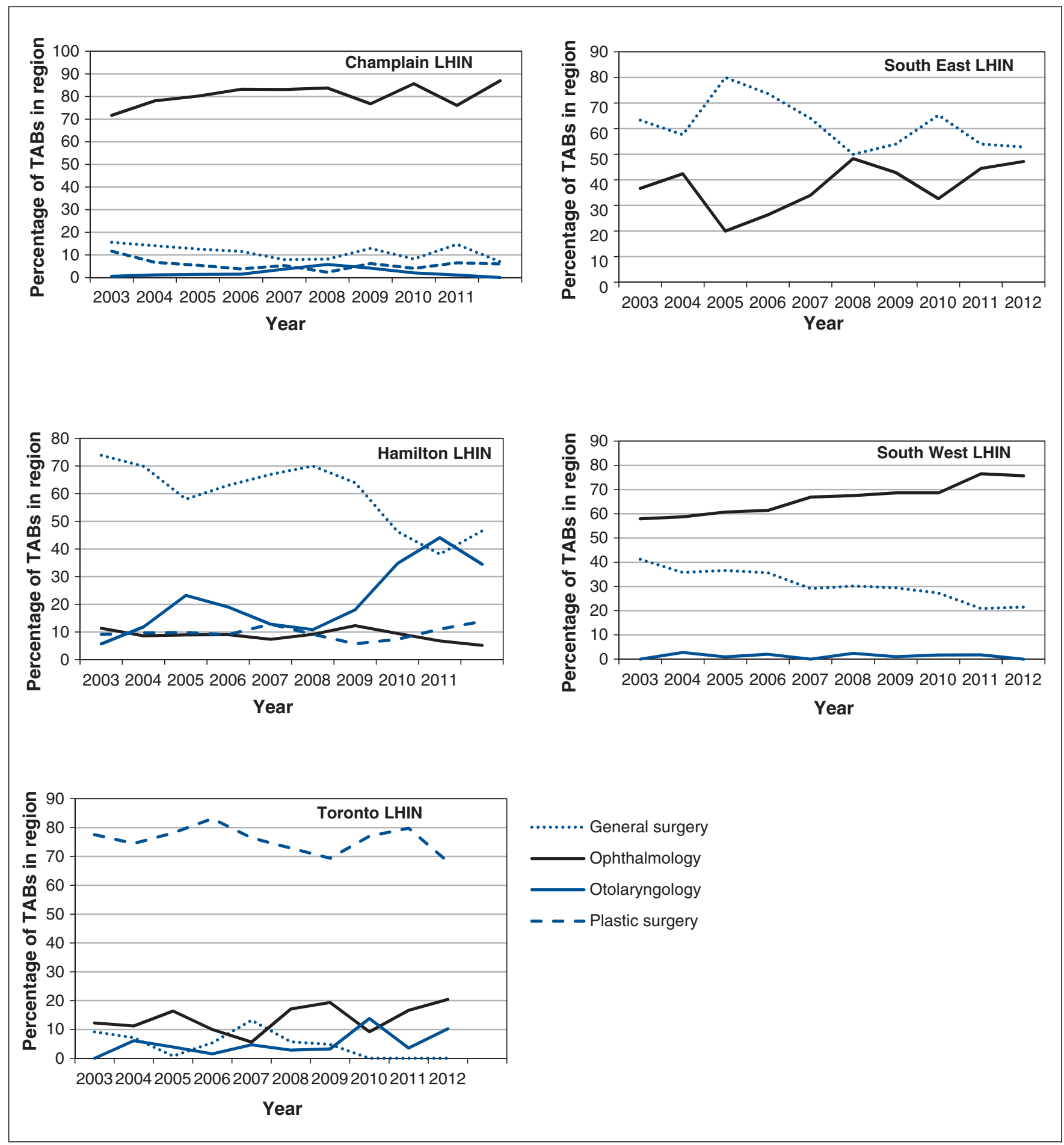

Figure 3: Proportion of all temporal artery biopsies (TABs) performed by region containing an ophthalmology residency program. Champlain = University of Ottawa, Ottawa, Ont.; LHIN = Local Health Integration Network; Hamilton = McMaster University, Hamilton, Ont.; South East = Queen's University, Kingston, Ont.; South West = Western University, London, Ont.; Toronto = University of Toronto, Toronto, Ont. 
involved in the diagnosis and treatment of giant cell arteritis did not recommend the use of temporal artery biopsy for diagnosis. Whether this trend is increasing or decreasing is not known. Finally, the number of true cases of giant cell arteritis may be stable, but physicians may be more selective about which patients should undergo temporal artery biopsy. This would be reflected in a greater rate of positive temporal artery biopsies over time.

The regional variation in the types of specialists performing temporal artery biopsies has implications for residency training. Exposure to the temporal artery biopsy procedure varied significantly by geographic location and areas with residency training programs. Graduates of certain residency programs may have had inadequate surgical volume in the area during their residency. This may leave some graduates unable to serve the community in which they practise in the future. It is important that residents be familiar with this geographic variation when referring suspected cases for temporal artery biopsy during their training. Surgical residents may want to seek out specialists comfortable with the procedure to perform a temporal artery biopsy when a patient suspected to have giant cell arteritis presents to a clinic or emergency department rather than referring it to another department. Moreover, in regions where few biopsies are done by a particular specialty, increasing exposure to the specialty that performs most of the temporal artery biopsies in the region during off-service resident rotations (in the internship year) may help improve the skills of graduating residents. Program directors, especially in general surgery, ophthalmology and plastic surgery, should be aware of the importance of their specialty at the population level and develop strategies to ensure their residents are competent in this area.

Much of the regional variation in the specialties performing temporal artery biopsies may be accounted for by the availability of surgeons familiar with the procedure. General surgeons performed the bulk of temporal artery biopsies in rural areas but performed only a small number per year. This also may have been due to a paucity of plastic surgeons, ophthalmologists or otolaryngologists in the area. ${ }^{14}$ Other factors that may have contributed to this variation are the availability of support staff, such as nurses and administration, and available space and equipment. In addition, financial remuneration may influence which procedures the specialists decide to perform at high volumes, especially if the additional costs of equipment and support staff must be incurred by the physician.

\section{Limitations}

To consider geographic location, Local Health Integration Units were used to divide regions in Ontario. These geographic boundaries are fluid rather than fixed, and the current study was limited in that travel across boundaries to receive care was not accounted for. In addition, only 1 temporal artery biopsy per person was included in the study, and bilateral biopsies were not considered. Some physicians elect to simultaneously perform 2 biopsies per person at the initial visit rather than reassess after the first result. Finally, only temporal artery biopsies that were billed to the Ontario
Health Insurance Plan were included and, if some residents and fellows performed this procedure without supervision or submitting the file for billing, the number of procedures performed may have been underestimated.

\section{Conclusion}

At the population level, general surgery, ophthalmology and plastic surgery have emerged as the primary specialties performing temporal artery biopsies in Ontario. The proportion of procedures performed by each specialty varied significantly by geographic location. Directors of residency programs should be aware of the role their specialty plays in regard to this procedure and develop new strategies to improve exposure to temporal artery biopsies in their region if it is lacking. Finally, the total number of patients undergoing temporal artery biopsy per 100000 population over 50 years of age has declined over the past decade and may reflect changes in the incidence of giant cell arteritis or changes in practice patterns. Future studies should aim to address the value different practitioners place on temporal artery biopsy and referral trends in Canada.

\section{References}

1. Danesh-Meyer HV. Temporal artery biopsy: skip it at your patient's peril. Am 7 Ophthalmol 2012;154:617-9.e1.

2. Dummer W, Zillikens D, Schulz A, et al. Scalp necrosis in temporal (giant cell) arteritis: implications for the dermatologic surgeon. Clin Exp Dermatol 1996; 21:154-8.

3. Slavin ML. Brow droop after superficial temporal artery biopsy. Arch Ophthalmol 1986;104:1127.

4. Bhatti MT, Goldstein MH. Facial nerve injury following superficial temporal artery biopsy. Dermatol Surg 2001;27:15-7.

5. Haist SA. Stroke after temporal artery biopsy. Mayo Clin Proc 1985;60:538.

6. Micieli JA. Geographic distribution of ophthalmologists in Ontario: a 10-year review. Can 7 Ophthalmol 2014;49:283-6.

7. Micieli JA, Micieli A, Schlenker MB, et al. Growth of medical and surgical vitreoretinal subspecialization in Ontario over 12 years. Can 7 Ophthalmol 2014;49:312-9.

8. Williams, JI, Young, W. A summary of studies on the quality of health care administration databases in Canada. In: Goel V, Williams JI, Anderson GM, et al., editors. Patterns of health care in Ontario: the ICES practice atlas. 2 nd ed. Ottawa: Canadian Medical Association; 1996:339-45. Available: www.ices.on.ca / /media/files/atlases-reports/1996/patterns-of-health-care-in-ontario-2nd-edition /Full\%20report.ashx (accessed 2015 Apr. 24).

9. Canada's ethnocultural mosaic, 2006 Census: provinces and territories. Ottawa: Statistics Canada; 2006. Available: www12.statcan.ca/census-recensement /2006/as-sa/97-562/p13-eng.cfm (accessed 2015 Apr. 24).

10. Study: Canada's visible minority population in 2017. Ottawa: Statistics Canada; 2005. Available: www.statcan.gc.ca/daily-quotidien/050322/dq050322b-eng. htm (accessed 2015 Apr. 24).

11. Pereira LS, Yoon MK, Hwang TN, et al. Giant cell arteritis in Asians: a comparative study. Br 7 Ophthalmol 2011;95:214-6.

12. Liu NH, LaBree LD, Feldon SE, et al. The epidemiology of giant cell arteritis: a 12-year retrospective study. Ophthalmology 2011;108:1145-9.

13. Drehmer TJ, Khanna D, Markert RJ, et al. Diagnostic and management trends of giant cell arteritis: a physician survey. 7 Rheumatol 2005;32:1283-9.

14. Iglesias S, Tepper J, Ellehoi E, et al. Rural surgical services in two Canadian provinces. Can 7 Rural Med 2006;11:207-17.

Affiliations: Department of Ophthalmology and Vision Science (J.A. Micieli, Margolin), University of Toronto; Faculty of Science (R. Micieli), York University, Toronto, Ont.

Contributors: All authors conducted the study and contributed to its design. Jonathan Micieli and Robert Micieli collected and managed data. All of the authors analyzed and prepared the data, prepared, reviewed and approved the final version to be published, and agreed to act as guarantors of the work.

Supplemental information: For reviewer comments and the original submission of this manuscript, please see www.cmajopen.ca/content/3/3/ E281/suppl/DC1 